\title{
Novel sequences of subgroup J avian leukosis viruses associated with hemangioma in Chinese layer hens
}

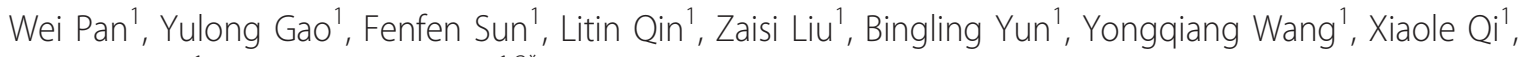
Honglei Gao ${ }^{1}$ and Xiaomei Wang ${ }^{1,2^{*}}$

\begin{abstract}
Background: Avian leukosis virus subgroup J (ALV-J) preferentially induces myeloid leukosis (ML) in meat-type birds. Since 2008, many clinical cases of hemangioma rather than ML have frequently been reported in association with ALV-J infection in Chinese layer flocks.

Results: Three ALV-J strains associated with hemangioma were isolated and their proviral genomic sequences were determined. The three isolates, JL093-1, SD09DP03 and HLJ09MDJ-1, were 7,670, 7,670, and 7,633 nt in length. Their gag and pol genes were well conserved, with identities of $94.5-98.6 \%$ and $97.1-99.5 \%$, respectively, with other ALV-J strains at the amino acid level (aa), while the env genes of the three isolates shared a higher aa identity with the env genes of other hemangioma strains than with those of ML strains. Interestingly, two novel 19-bp insertions in the U3 region in the LTR and 5' UTR, most likely derived from other retroviruses, were found in all the three isolates, thereby separately introducing one E2BP binding site in the U3 region in the LTR and RNA polymerase II transcription factor IIB and core promoter motif ten elements in the $5^{\prime}$ UTR. Meanwhile, two binding sites in the U3 LTRs of the three isolates for NFAP-1 and AIB REP1 were lost, and a 1-base deletion in the E element of the 3' UTR of JL093-1 and SD09DP03 introduced a binding site for c-Ets-1. In addition to the changes listed above, the rTM of the $3^{\prime}$ UTR was deleted in each of the three isolates.
\end{abstract}

Conclusion: Our study is the first to discovery the coexistence of two novel insertions in the U3 region in the LTR and the 5' UTR of ALV-J associated with hemangioma symptoms, and the transcriptional regulatory elements introduced should be taken into consideration in the occurrence of hemangioma.

Keywords: Avian leukosis virus subgroup J, Layer, Hemangiomas, Sequence analysis, Transcriptional regulatory elements

\section{Background}

Avian leukosis virus subgroup J (ALV-J), the most recently discovered avian exogenous retrovirus, is thought to have emerged via a recombination event between an unknown exogenous ALV and an endogenous retrovirus [1-3]. Since the first report of the prototype ALV-J strain HPRS-103 in 1989 in the United Kingdom $[1,4,5]$, the virus has been found worldwide, predominantly in meat-type chickens [5]. In China,

\footnotetext{
* Correspondence: xmw@hvri.ac.cn

'Division of Avian Infectious Diseases, State Key Laboratory of Veterinary Biotechnology, Harbin Veterinary Research Institute, Chinese Academy of Agricultural Sciences, Harbin 150001, PR China

Full list of author information is available at the end of the article
}

ALV-J infection of broilers was first detected and officially recognized in 1999 [6], followed by scattered reports of infection of broiler and local chickens in some areas of China [7-9]. Although egg-type chickens have been experimentally infected with ALV-J to induce tumors [10], no field cases of ALV-J infection and tumors in commercial layer chickens were found in China until 2004 [11]. Curiously, ALV-J infection in Chinese layer flocks has become widespread since 2008. Cases of ALV-J, infection and tumors in commercial layer chickens have been emerging in China in recent years, causing severe production problems in layer flocks [12-15].

\section{C) Biomed Central}


Clinical infection with ALV-J is associated with the development of various tumors such as myelocytomas (ML), erythroblastosis (EB), hemangiomas, nephromas, and sarcomas $[16,17]$. ML is the most commonly reported neoplastic syndrome associated with ALV-J in broiler chickens [17]. However, the dominant tumor induced by ALV-J infection in Chinese layer chickens is hemangioma. Large numbers of clinical hemangioma cases in egg-type chickens have been reported in China [7,12-15]. Hemangiomas are vascular tumors characterized by the abnormal growth of endothelial cells from capillary blood vessels [18]. This type of tumor may be induced in chickens by an avian leukosis retrovirus (ALV) variant that contains the erb-B oncogene [19]. It seems that the ALV-Js common in the field induce a greater spectrum of tumors because ML has also occurred in some hemangioma cases $[7,14]$. Whether the change in the tumor spectrum of ALV-J in chickens is related to the sequence variation of this virus or the species of the host is still unknown.

To better understand the molecular characteristics of ALV-J, three hemangioma isolates, JL093-1, SD09DP03 and HLJ09MDJ-1, isolated from three separate provinces in China, were first confirmed to be associated with ALV-J. Then, the whole genomic sequences of the three isolates were determined and compared with the published sequences of ALV-J strains, including hemangioma and ML strains. Interestingly, two novel 19-bp insertions in the U3 LTR and the 5' UTR, most likely derived from other retroviruses, were found in all the three isolates. These insertions introduced one E2BP binding site in the U3 LTR and RNA polymerase II transcription factor IIB and core promoter motif ten elements in the 5' UTR. Meanwhile, the binding sites for NFAP-1 and AIB REP1 in the U3 LTR of the three isolates were lost, and a 1-base deletion in the E element of the 3' UTR of JL093-1 and SD09DP03 introduced a binding site for c-Ets-1. These transcriptional regulatory elements, known as factors associated with vascular tumors [20-23], were speculated to be related to the development of hemangioma. Herein, we report for the first time the coexistence of two $19 \mathrm{bp}$ insertions in a hemangioma ALV-J.

\section{Methods}

\section{Clinical samples}

In late 2009, suspected cases of ALV-J infection erupted in layer flocks in three main chicken-industry provinces (Jilin, Shandong, Heilongjiang) of China. The levels of egg production were dramatically reduced in the affected flocks. Clinical symptoms included hemorrhages in the skin of the phalanges and feather follicles. Some layer hens had gray-white nodules in the liver, spleen or kidneys, and the liver and spleen were enlarged up to several times their normal size. Morbidity in some flocks reached $60 \%$, and the mortality rate was over $20 \%$, causing heavy economic losses. To confirm and analyze the pathogenesis of this disease, livers, spleens, and tumors were collected from sick chickens in the three provinces.

\section{Virus isolation}

The diseased tissues or tumors were inoculated onto the DF-1 cells, and incubated at $37^{\circ} \mathrm{C}$ with $5 \% \mathrm{CO} 2$ for five days for each passage [24]. Uninfected DF-1 cells were used as negative control. After one blind passage, the existence of ALV-J in DF-1 cells was verified by PCR detection of 545 bp repeated sequence [25]. Three blind passage were conducted until the result of PCR detection of cellular genome and RT-PCR detection of supernatant were both positive.

\section{Primers}

Four primers pairs were designed to amplify in the whole genomic sequences of ALV-J isolates (Table 1). Primer pairs W1, W2, and W3 were used to amplify the sequence from position 1 - 7841 of the prototype ALV-J strain HPRS-103, and primer pair W4 was used to amplify the long terminal repeat (LTR) sequences from circularized ALV-J viral DNA.

\section{Genomic DNA extract and PCR amplification}

The total DNA was extracted from DF-1 cells infected with virus by using a sodium dodecyl sulfate (SDS) proteinase $\mathrm{K}$ and phenol/chloroform/isoamylol (25:24:1) protocol. Genomic DNA PCR amplification was performed according to the manual of rTaq kit (Takara, Dalian, China), by using the proviral genomic DNA as template. The optimum conditions for PCR were as follows: $95^{\circ} \mathrm{C}$ for $5 \mathrm{~min}, 30$ cycles at $95^{\circ} \mathrm{C}$ for $30 \mathrm{~s}, 55^{\circ} \mathrm{C}$ for $30 \mathrm{~s}, 72^{\circ} \mathrm{C}$ for $2 \mathrm{~min} 40 \mathrm{~s}$, and a final elongation at $72^{\circ} \mathrm{C}$ for $10 \mathrm{~min}$. The PCR product was analyzed in $1 \%$

\begin{tabular}{|c|c|c|c|}
\hline Segments & Primers & Sequences(from 5'- 3') & Product sizes/bp \\
\hline \multirow[t]{2}{*}{ W1 } & W1F & GGTGTAGTGTTATGCAATACTC & 2782 \\
\hline & W1R & ACAGGCGTGTGGTCTGGCTTCC & \\
\hline \multirow[t]{2}{*}{ W2 } & W2F & GGACTGTTGCGCTACATCTGGC & 2788 \\
\hline & W2R & GACCCACACGTTTCCTGGTTG & \\
\hline \multirow[t]{2}{*}{ W3 } & W3F & GTGCGTGGTTATTATTTCCGTT & 2330 \\
\hline & W3R & ACCAATGTGGTGGGAGGTAAA & \\
\hline \multirow[t]{2}{*}{ W4 } & W4F & TTAGGAAGGCAACAGACGG & 466 \\
\hline & W4R & GGGCGACCAGAATCACG & \\
\hline
\end{tabular}

Primers W1, W2, W3 used to amplify the sequence from position 1 - 7841 of the prototype ALV-J strain HPRS-103. Primers W4 used to amplify the long terminal repeat (LTR) sequences from circularized viral DNA of the three isolates 
agarose in Tris-borate - EDTA (TBE) buffer gel containing $0.5 \mathrm{mg} / \mathrm{ml}$ ethidium bromide.

\section{Cloning and sequencing of proviral genomic DNA}

All PCR productions were then cloned into the TA vector PMD18-T (TAKARA, Biotechnology Co., Ltd., Dalian, China), and they were sequenced with an $A B$ 3730 DNA sequencer by commercial services in China. Due to the possibility of genetic variation, at least three independent plasmids were sequenced for confirmation.

\section{Sequence analysis}

To analyze the sequences of hemangioma ALV-J isolates, the sequences of reference ALV-J strains, including the sequences of all published ML and other hemangioma ALV-Js, were obtained from GenBank. The accession numbers are listed in Table 2. Multiple sequence alignment was carried out using the sequence analysis software Lasergene 1 (DNASTAR Inc., Madison, WI) and Clustal X 1.83 [26]. An unrooted phylogenetic tree was generated by the distance-based neighbor-joining method using MEGA 4.1 [27]. Bootstrap values were calculated using 500 or 1000 replicates of the alignment. Transcriptional regulatory elements in the U3 were analyzed by NSITE (Recognition of Regulatory motifs), an online service of Soft Berry (http://linux1.softberry.com/ berry.phtml).

\section{Results}

Virus isolation and identification

Three ALV-J strains were isolated from the sick chickens, and were designated as JL093-1, SD09DP03, and

Table 2 ALV-J reference strains and other retroviruses used in this study

\begin{tabular}{|c|c|c|c|c|c|}
\hline Strains & Year & Country & Origin host & Tumor type & Accession No. \\
\hline HPRS-103 & 1995 & UK & white broiler & $\mathrm{ML}$ & Z46390 \\
\hline ADOL-7501 & 2001 & USA & white broiler & $M L$ & AY027920 \\
\hline $\mathrm{NX0101^{a }}$ & 2001 & China & white broiler & $M L$ & DQ115805 \\
\hline YZ9902 & 1999 & China & white broiler & $M L$ & HM235670 \\
\hline SD07LK1 ${ }^{a}$ & 2007 & China & commercial layer & $M L$ & FJ216405 \\
\hline$J S-n t^{a}$ & 2003 & China & white broiler & $M L$ & HM235667 \\
\hline $\mathrm{NHH}^{\mathrm{a}}$ & 2007 & China & commercial layer & $\mathrm{He}$ & HM235668 \\
\hline $\operatorname{SCDY} 1^{a}$ & 2009 & China & parental layer & $\mathrm{He}$ & HQ425636 \\
\hline JS09GY3 ${ }^{a}$ & 2009 & China & commercial layer & $\mathrm{He}, \mathrm{ML}$ & GU982308 \\
\hline JS09GY6 & 2009 & China & commercial layer & $\mathrm{He}, \mathrm{ML}$ & GU982310 \\
\hline NM2002-1 ${ }^{a}$ & 2002 & China & white broiler & $\mathrm{ML}$ & HM235669 \\
\hline HLJ09MDJ-1 & 2009 & China & helan layer & $\mathrm{He}$ & JN624878 \\
\hline JL09JL3-1 & 2009 & China & commercial layer & $\mathrm{He}$ & JN624879 \\
\hline SD09DP03 & 2009 & China & commercial layer & $\mathrm{He}$ & JN624880 \\
\hline JL10HW02 & 2010 & China & commercial layer & $\mathrm{He}$ & HQ634801 \\
\hline HLJ10SH04c & 2009 & China & commercial layer & $\mathrm{He}$ & HQ634814 \\
\hline HuB09WH02 & 2009 & China & commercial layer & $\mathrm{He}$ & HQ634804 \\
\hline HuB09WH03 ${ }^{c}$ & 2010 & China & commercial layer & $\mathrm{He}$ & HQ634805 \\
\hline HLJ10SH01 ${ }^{c}$ & 2010 & China & commercial layer & $\mathrm{He}$ & HQ634806 \\
\hline HLJ10SH03 & 2010 & China & commercial layer & $\mathrm{He}$ & HQ634813 \\
\hline HLJ10SH02 & 2010 & China & commercial layer & $\mathrm{He}$ & HQ634807 \\
\hline HN1001-1 ${ }^{c}$ & 2010 & China & commercial layer & $\mathrm{He}$ & HQ260974 \\
\hline HN1001-2 & 2010 & China & commercial layer & $\mathrm{He}$ & HQ260975 \\
\hline HN1001-3c & 2010 & China & commercial layer & $\mathrm{He}$ & HQ260976 \\
\hline PR2257 & 1989 & Czechoslovakia & unknow & sarcoma & L21974 \\
\hline RSV-SRA ${ }^{b}$ & 1995 & USA & unknow & sarcoma & U41731 \\
\hline $1 C 4^{b}$ & 1991 & France & unknow & $\mathrm{L}$ & X77628 \\
\hline $\mathrm{RAV}-1^{\mathrm{b}}$ & 1990 & France & unknow & $L$ & M62407 \\
\hline$R S V-S R B^{b}$ & 1998 & USA & unknow & sarcoma & AF052428 \\
\hline$\left.\mathrm{HBl}\right|^{b}$ & 1984 & Germany & unknow & $\mathrm{L}$ & M11784 \\
\hline$R A V-1^{b}$ & 1990 & France & unknow & $\mathrm{L}$ & M62407 \\
\hline
\end{tabular}

${ }^{\mathrm{a}}$ strains can obtain the whole sequences from GenBank.

${ }^{\mathrm{b}}$ strains are other retroviruses published.

cstrains only env gene available in GenBank.

$M L$ means myeloma leukosis, He represents hemangioma, and $L$ remarks lymphoid tumors 
HLJ09MDJ-1. The genomic DNA of infected DF-1 cells were positive in PCR detection, and the supernatant of infected DF-1 cells was positive in RT-PCR detection (data not shown), indicating the existence of proviral genomic DNA of ALV-J in infected DF-1 cells.

\section{Sequence comparison of the three isolates and other hemangioma and ML strains}

The whole genome sequences of the three isolates were aligned with DNASTAR (version 5.01), and the sequences were submitted to GenBank with the accession numbers as follows: HLJ09MDJ-1 (JN624878), SD09DP03 (JN624880), and JL093-1 (JN624879). The full-length proviral genome sequences of JL093-1, SD09DP03 and HLJ09MDJ-1 were 7670, 7670 and 7633 nt in length, respectively. To better characterize ALV-Js associated with hemangioma on a molecular level, we compared the whole sequences of the three isolates with the sequences of four other hemangioma strains and eight ML strains published in GenBank (Table 2).

The homology analysis of gag, pol and env genes of the three isolates

Comparisons of the three major genes revealed that the homology of nucleotide sequence of pol and gag genes between the three isolates and other isolates in GenBank were $94.5-98.6 \%, 97.1-99.5 \%$ at the amino acid (aa) level, respectively. These data suggested that the pol and gag genes of all ALV-Js were highly conserved.

However, hemangioma and ML ALV-Js seem to have different env genes. env genes of the three isolates and other hemangioma isolates was $88.5-91.7 \%$ identical to that of ML strains at the aa level, but a much higher identity (92.7-99.6\%) was found with all hemangioma strains (except for HLJ09MDJ-1 and NHH), which may be indicative of the close relationship among hemangioma isolates. Phylogenetic analysis demonstrated that hemangioma isolates belonged to the same branch and that the reference ML strains clustered in the other branch (Figure 1C).

Novel sequences in LTRs and 5'UTRs of the three isolates A 19-bp insertion (5' CTGTAGTCTTGCAACATGC 3'), located between sites 19 and 20 according to the sequence of the prototype ALV-J strain HPRS-103, occurred in the U3 region in the LTR of the three isolates (Figure 2). This insertion is essentially also identical to sequences found in some other retroviruses, including human hamster virus PR2257 and Rous sarcoma virus strain Schmidt-Ruppin A (RSV-SRA). NSITE, an online service provided by Soft Berry, showed that some transcriptional regulatory elements of the U3 region of the three isolates had changed (Figure 2). In

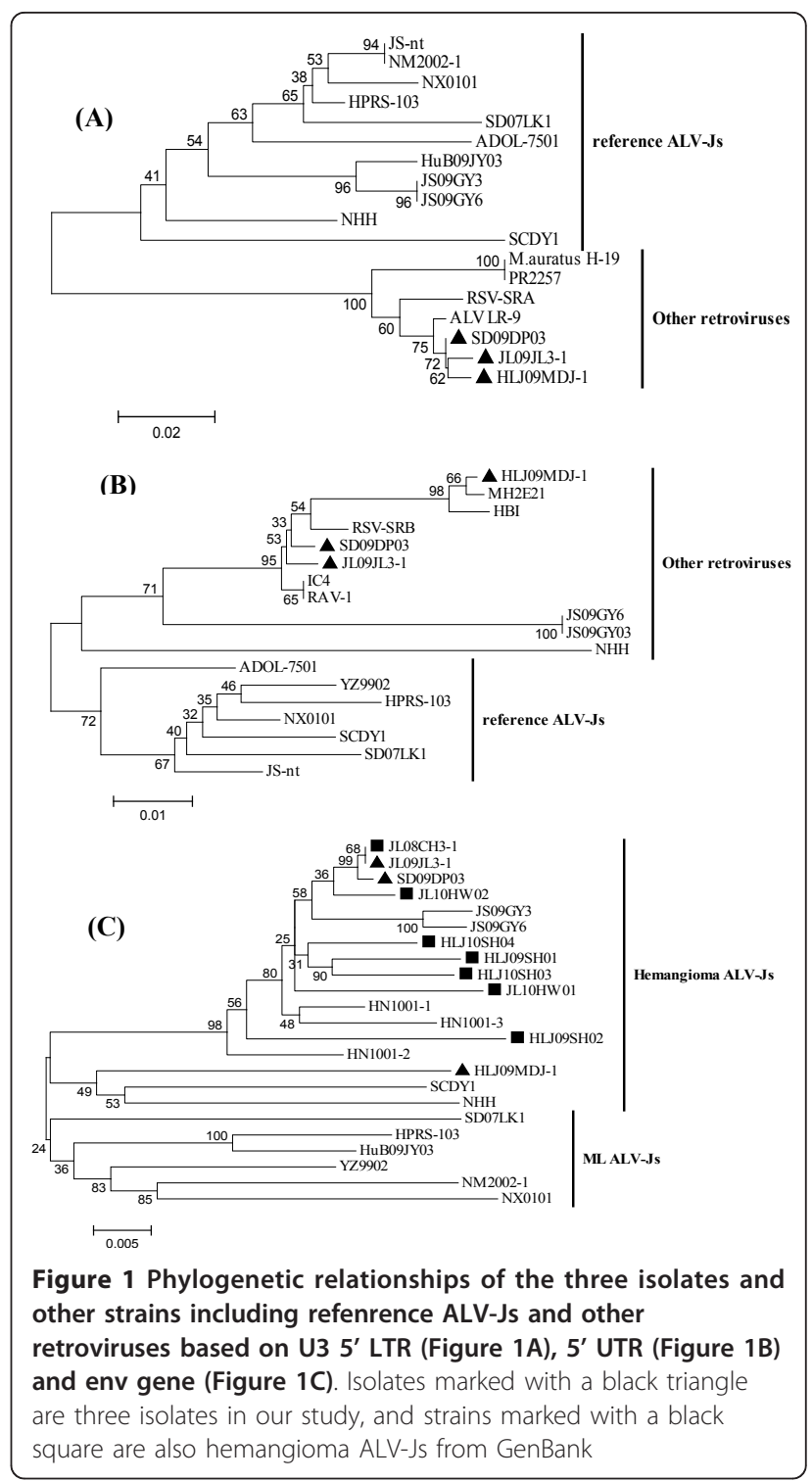

contrast with ML strains, in the three isolates, the NFAP-1 and AIB REP1 components were lost due to the mutation and deletion of base pairs, which was also observed in the hemangioma strain SCDY1 [12]. An E2BP binding site was introduced by the 19 bp-insertion. Other components, including a C/EBP, two CArG boxes, two Y boxes, and one TATA box, were still well conserved. Homology analysis showed that the U3 region in the LTRs of the three isolates shared 91.8$95.3 \%$ homology with those of other retroviruses, but only 81.7-87.1\% similarity with ML ALV-Js. The U3 sequences of the three isolates clustered in a distinct group apart from homologous sequences, while other hemangioma strains such as SCDY1 and NHH belonged to the same branch as ML strains in the phylogenetic tree (Figure 1A). 


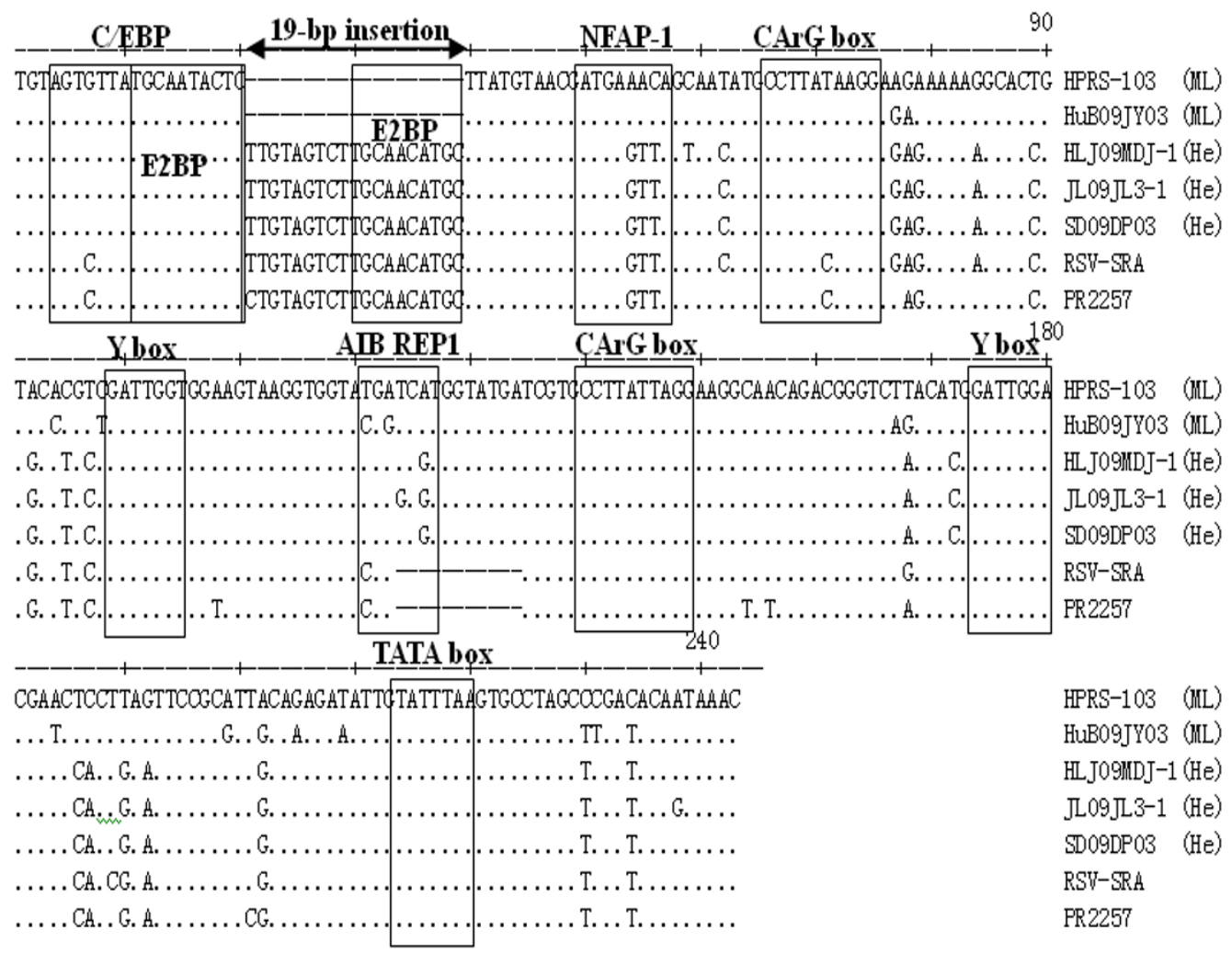

Figure 2 Comparision of transcriptional regulatory elements in the U3 region of reference strains. Shown are the sequences of U3 from HPRS-103 [5]. Sequences identical to HPRS-103 are showed as dot (.) and the deletions are represented by dashes (-).The 19 bp novel insertion in the three isolates is marked and transcriptional regulatory elements in U3 area are boxed

Another 19-bp sequence (5' TGCTCTGCGTGATTCCGGT 3'), located between sites 259 and 260 according to 5 ' UTR of HPRS-103, was found in the 5' UTRs of the three isolates; this insertion is also present in the hemangioma strain JS09GY6 but is not present in any ML ALV-J strain published (Figure 3). Interestingly, the 19-bp insertion was predicted to introduce RNA polymerase II transcription factor II B and core promoter motif ten elements, which are also present in two hemangioma strains, SCAU-HN06 and JS09GY6 [13,14]. Homology analysis revealed that the 5' UTRs of the three isolates were closely associated with those of type 1 Rouse sarcoma related virus (RAV-1), Rous sarcoma virus strain SchmidtRuppin A (RSV-SRB) and other retroviruses, sharing 97.5$100 \%$ identity with each other. Phylogenetic analysis also demonstrated that all hemangioma ALV-Js and other retroviruses belonged to the same branch and that, strikingly, the published ML strains were present in the other branch (Figure 1B).

The molecular features of the 3' UTRs of the three isolates

Three elements, the redundant transmembrane region (rTM), the direct repeat 1 (DR 1) and the E element, are located in the 3' UTR. The molecular characterization of the 3' UTR of the three isolates was as follows.

Deletion of most of the rTM Consistent with ML strains, most of the rTMs of the three isolates were deleted, as observed in other hemangioma strains such as SCAU-HNO6 and JS09GY6 [13,14] and the ML strain ADOL-7501. The rTM was found only in earlier ML strains such as HPRS-103 [1]. The deletion of the rTM is common in current ALV-J isolates, suggesting that this region might be dispensable for viral fitness $[13,14]$.

Complete conservation of DR 1 As a single or double copy element flanking the src in some oncogenic sarcoma viruses, DR 1 had been found exclusively in sarcoma viruses and ALV-J in the past [1]. To our knowledge, DR 1 is present in the 3' UTR of all ALV-J isolates, without any exception. DR 1 may contribute to the fitness of the viruses that contain them because the element is associated with the efficient accumulation of unspliced RNA in the cytoplasm and the selective increase in the amount of spliced src mRNA in ALV.

A binding site for c-Ets-1 was introduced in the $\mathrm{E}$ element Most base pairs of the E element were conserved in the three hemangioma isolates, but minor mutations were observed (Figure 4). For example, the E 


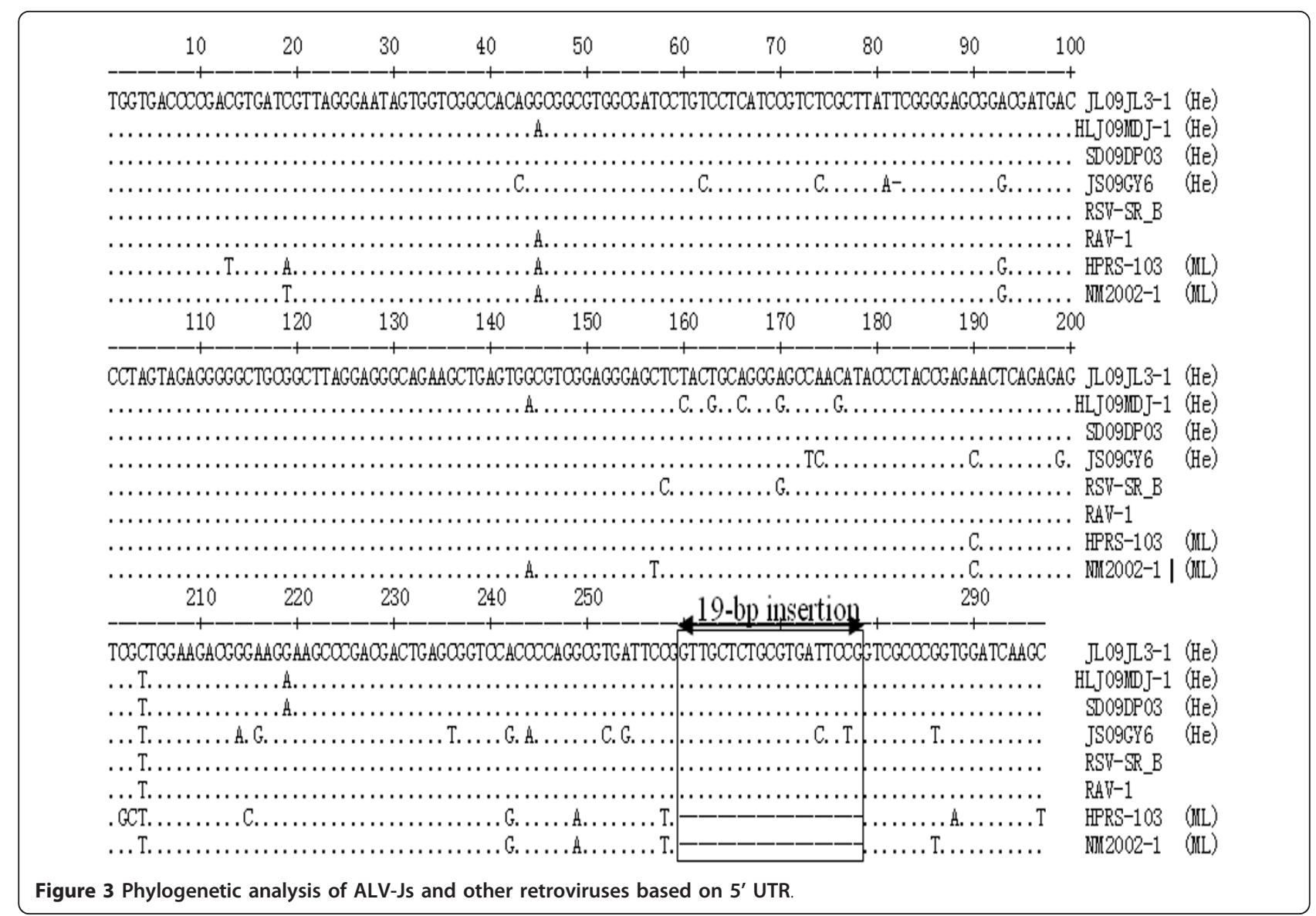

elements of JL093-1 and SD09DP03 had a 1-base deletion (between 7387 and 7389 according to the HPRS103 sequence); this deletion was also present in JS09GY6, JS09GY3, JS09GY5 and SCAU-HN06 [13,14].
The deletion introduced a specific distinct binding site for c-Ets-1 according motif analysis; this binding protein is associated with vascular endothelial cell differentiation. In contrast, most parts of the E element of ML

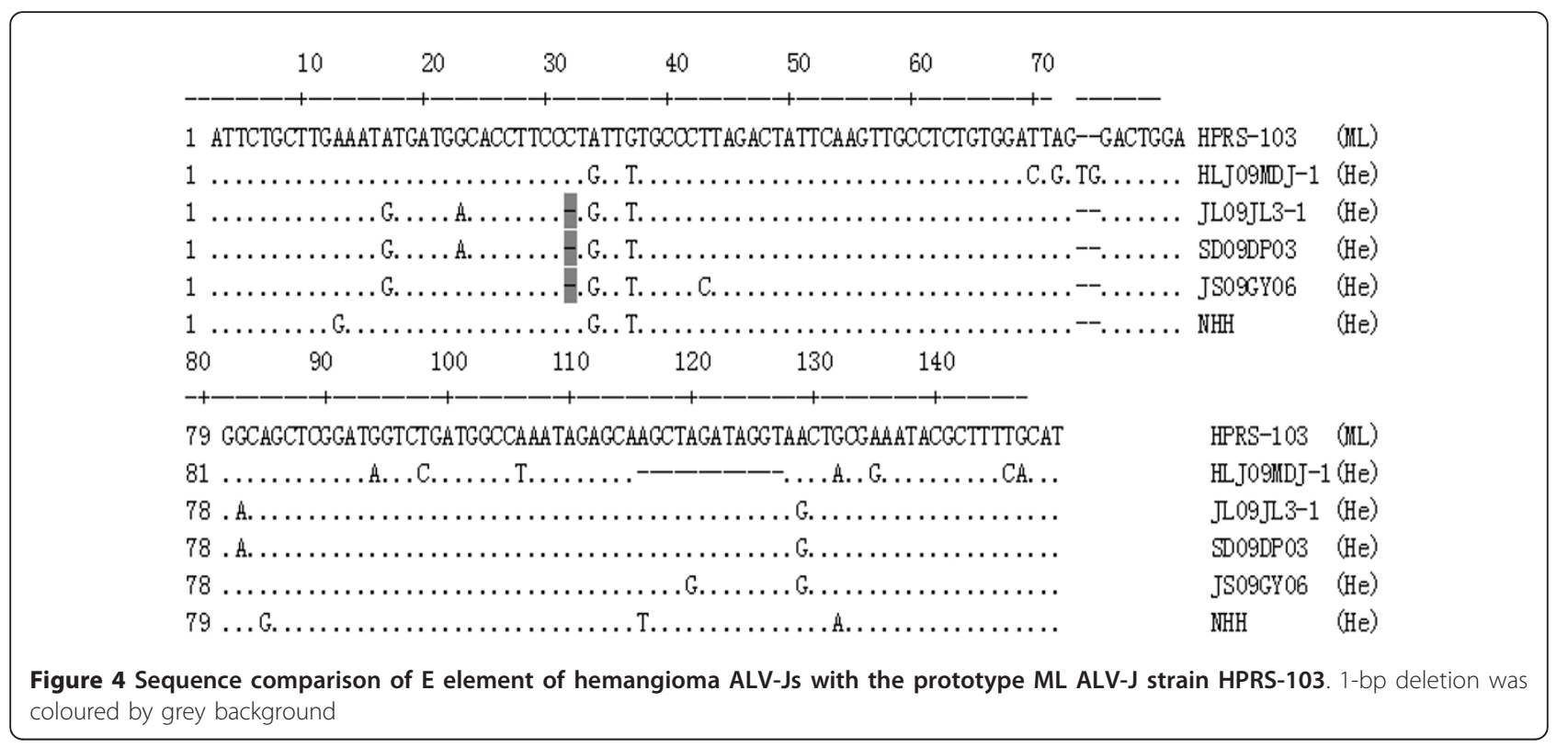


strains whose sequences have been reported to date have been deleted. For example, in the USA, the E element of ML strains have been reported to have substantial deletions in $>50 \%$ of ALV-Js [28], and the E element has been completely deleted in earlier Chinese ML isolates such as YZ9902 and NX0101 and the recent strain JS-nt.

\section{Discussion}

In this study, the whole genomic sequences of three ALV-J isolates associated with hemangioma from layers were determined and compared with the published sequences of $M L$ and hemangioma ALV-J strains. Although the three isolates were collected from different sick layers in three separate provinces of China, they all contained special sequences such as modifications of the LTRs and 5' UTRs that seem to be molecular markers of some hemangioma strains.

Consistent with other ALV-J strains, gag and pol genes of the three isolates were highly conserved. However, env genes of hemangioma strains seem to differentiate from ML strains, since the fact that all hemangioma strains were clustered in the same branch while ML strains belonged to the other branch in the phylogenetic tree (Figure 1C). The env glycoprotein of $\mathrm{ALV}$, as in other retroviruses, functions mainly as a ligand for receptor binding for virus entry into susceptible cells [29], and was also demonstrated to be a major determinant of the lineage-specific oncogenicity and host range [30-32]. Whether the tumor spectrum is determined by the env genes of ALV-J or not needs further study.

Hemangioma ALV-Js seem to contain special U3 region in the LTR and 5' UTR sequences. Phylogenetic analyses based on the U3 LTR and the 5' UTR revealed that all hemangioma isolates examined and some other retroviruses belonged to the same branch, whereas the published ML ALV-J sequences belonged to another branch (Figure 1A, 1B), implying that these U3 LTR and the 5' UTR sequences of hemangioma ALV-Js were most likely derived from some other retroviruses. To our knowledge, the ALV-J strains reported to date have been recognized as slowly transforming viruses, while related retroviruses such as HBI and RSVs quickly induce tumors in chickens and have altered biological properties [33,34]. For example, HBI, which was derived from the myc-containing virus MC29, was demonstrated to be capable of inducing a variety of tumors when injected into newborn chickens [33]. Hence, the acutelike sequences of those hemangioma strains may influence the tumor spectrum and pathogenicity of ALV-J in layers. The three isolates in this study can replicate to a high titer in DF-1 cells, a characteristic that is shared with some other hemangioma viruses [29]. This new biological property may have a close relationship with the U3 of the 5' LTR and the 5' UTR contained in the genome of ALV-Js.

It is noteworthy that there were two 19 bp insertions in the U3 LTR and 5' UTR in our three isolates that have not been previously reported. The U3 LTR region of the avian retroviruses has been extensively characterized as a model of a strong transcription regulatory unit. This compact enhancer and promoter drives high levels of viral and cellular gene transcription in many cell types in birds and in mammals [35-37]. According to our analysis, parts of the transcriptional regulatory elements in the U3 region of the three isolates have changed. The binding sites for NFAP-1 and AIB REP1 were not observed in our three isolates but exist in all $\mathrm{ML}$ ALV-Js. The NFAP-1 site is recognized by activator protein 1 (AP-1). AP-1 is a heterodimeric protein that regulates gene expression in response to stimuli such as cytokines, growth factors, stress, and bacterial and viral infections [20]. AIB REP1, known as repair of chromatin damage 1 , was recently reported to be a regulatory factor of human gene Apo-AI. Apo-AI is closely related to the vascular invasion of hepatocellular carcinoma in humans [21]. The absence of the NFAP-1 and AIB REP1 binding sites may influence the infection abilities of the ALV-J virus in chickens or the development of hemangioma. Moreover, the insertion in the U3 introduced an E2BP binding site. E2BP was first found in hepatitis $B$ virus (HBV) and influences the liver specificity of HBV replication through the combination with an enhancer, EII [22]. The additional DNA binding site for E2BP introduced in the U3 may be related to viral tropism [23]. The insertion or deletion of transcription regulatory elements may severely impact the transcriptional activity of the LTR in hemangioma isolates, thereby affecting the replication or infection capability of this virus.

The 5' UTR played an important role in viral replication through potential intra- and intermolecular interactions [38]. One RNA polymerase II transcription factorIIB element and core promoter motif ten elements were introduced via a 19-bp insertion in the 5' UTR of all three isolates; this insertion is also found in the hemangioma strains SCAU-HN06 and JS09GY6 [13,14]. However, the influence of the inserted sequence in viral replication is still unknown.

Elements in the 3' UTR play a key role in virion assembly and the ability to induce tumors [39]. Early studies found that the rTM exists in the majority ALV-J trains in China; however, deletion of the rTM has been observed in a large number of ALV-J strains in recent years $[13,14,40]$. The loss of the rTM seems to be a trend in the sequence variation of the 3' UTR in current ALV-Js in China (unpublished data). Therefore, the 
rTM is suspected to be related to the evolution and virulence of this virus, and its role should not be ignored. The E element, containing a biding site for the transcription factor c/EBP and acting as an enhancer, was previously only found in the gene of sarcoma viruses [41]. Currently, the E element is present in many ALV-J strains in addition to our isolates (unpublished data). The existence of the E element in the 3'UTR does increase the occurrence rate of tumors in chickens infected with ALV-J, although this element is not the decisive factor in the induction of tumors [42]. For the hemangioma ALV-Js examined, a binding site for c-Ets1, introduced in JL093-1 and SD09DP03 due to a 1-bp deletion, was associated with the development of hemangiomas. Consistent with other reports, DR 1 of all hemangioma ALV-Js was very well conserved. This high level of conservation is consistent with the fact that DR 1 plays an important role in the assembly of the genomic RNA of ALV-Js [43].

In conclusion, our study is the first to discovery the coexistence of two novel insertions in the U3 region in the LTR and the 5' UTR of ALV-J associated with hemangioma symptoms, and the transcriptional regulatory elements introduced should be taken into consideration in the occurrence of hemangioma.

\section{Acknowledgements}

The study was supported by the National Natural Science Foundation of China (31072146), the earmarked fund for the Modern Agro-industry Technology Research System (No. nycytx-42-G3-01), and the Harbin Programs for Science and Technology Development (No. 2010AA6AN034).

\section{Author details}

'Division of Avian Infectious Diseases, State Key Laboratory of Veterinary Biotechnology, Harbin Veterinary Research Institute, Chinese Academy of Agricultural Sciences, Harbin 150001, PR China. ${ }^{2}$ Harbin Veterinary Research Institute, 427 Maduan Street, Harbin 150001, PR China.

\section{Authors' contributions}

WP carried out the molecular genetic studies, participated in the sequence alignment and drafted the manuscript. YLG guided the study design, and revised the manuscript. FFS, LTQ, BLY, ZSL, YQW, XLQ, HLG helped in experiments. XMW participated in its design and coordination and helped to draft the manuscript. All authors read and approved the final manuscript.

\section{Competing interests}

The authors declare that they have no competing interests.

Received: 30 August 2011 Accepted: 21 December 2011 Published: 21 December 2011

\section{References}

1. Payne LN, Brown SR, Bumstead N, Howes K, Frazier JA: A novel subgroup of exogenous avian leucosis virus in chickens. J Gen Virol 1991, 72:801-807.

2. Smith LM, Toye AA, Howes K, Bumstead N, Payne LN, Venugopal K: Novel endogenous retroviral sequences in the chicken genome closely related to HPRS-103(subgroup J) avian leukosis virus. J Gen Virol 1999, 80:261-268.

3. Tsichlis PN, Donehower L, Hager G, Zeller N, Malavarca R, Astrin S: Sequence comparison in the crossover region of an oncogenic avian retrovirus recombinant and its nononcogenic parent: genetic regions that control growth rate and oncogenic potential. Mol Cell Biol 1982, 2:1331-1338.

4. Payne LN, Howes K, Gillespie AM: Host range of Rous sarcoma virus pseudotye RSV (HPRS-103) in 12 avian species: support for a new avian retrovirus gp85elope subgroup. J Gen Virol 1992, 73:995-997.

5. Bai J, Payne LN, Skinner MA: HPRS-103 (exogenous avian leukosis virus, subgroup J) has an env gene related to those of endogenous elements EAV-0 and E51 and an E element found previously only in sarcoma viruses. J Virol 1995, 69:779-784.

6. Du Y, Cui ZZ, Qin AJ: Subgroup J of avian leukosis viruses in China. China Poult Sci 1999, 3:1-4.

7. Chen Z, Zhang L, Liu S, Zhang L, Cui Z: Emerging of avian leukosis virus subgroup $\mathrm{J}$ in a flock of Chinese local breed. Acta Microbiol Sin 2005, 45:584-587.

8. Sun SH, Cui ZZ: Epidemiological and pathological studies of subgroup avian leukosis virus infections in Chinese local "yellow"chickens. Avian Pathol 2007, 36:221-226.

9. Cheng Z, Liu J, Cui Z, Zhang L: Tumors associated with avian leukosis virus subgroup J in layer hens during 2007 to 2009 in China. J Vet Med Sci 2010, 72:1027-1033.

10. Payne LN, Gillespie AM, Howes K: Myeloid leukemia and transmission of the HPRS-103 strain of avian lekosis virus. Leukemia 1992, 6:1167-1176.

11. Xu B, Dong W, Yu C, He Z, LV Y, Sun Y: Occurrence of avian leukosis virus subgroup J in commercial layer flocks in China. Avian Pathol 2004, 33:13-17.

12. Min S, Ming-xing $T$, Cheng $L$, Yang $Z$, Yan L, Nian-li Z, Ping L, Yong $H$ : Sequence analysis for the complete proviral genome of subgroup $J$ avian leukosis virus associated with hemangioma: a special $11 \mathrm{bp}$ deletion was observed in U3 region of 3' UTR. Virol J 2011, 8:158.

13. Lai H, Zhang $H$, Ning Z, Chen R, Zhang W, Qin A, Xin C, Yu K, Liao M: Isolation and characterization of emerging subgroup J avian leukosis virus associated with hemangioma in egg-type chickens. Vet Microbiol 2011, 3:1-9.

14. Wu X, Qian K, Qin A, Wang P, Jin W, Eltahir YM: Recombinant avian leukosis viruses of subgroup $J$ isolated from field infected commercial layer chickens with hemangioma and myeloid leukosis possess an insertion in the E element. Vet Res Commun 2010, 34:619-632.

15. Gao $Y L$, Qin LT, Pan W, Wang YQ, Qi XL, Gao HL, Wang XM: Subgroup J avian leukosis virus in layer flocks in China. Emerg Infect Dis 2010, 16:1637-1638.

16. Arshad SS, Bland AP, Hacker SM, Payne LN: A low incidence of histiocytic sarcomatosis associated with infection of chickens with the HPRS-103 strain of subgroup J avian leukosis virus Avian leukosis virus. Avian Dis 1997, 41:947-956.

17. Stedman NL, Brown TP: Cardiomyopathy in broiler chickens congenitally infected with avian leukosis virus subgroup J. Vet Pathol 2002, 39:161-164.

18. Grant JW, Gallagher PJ, Hendinger C: Hemmangioblastoma. An immnohistochemical study of ten cases. Acta Neuropathol 1988, 76:82-86.

19. Tracy SE, Woda BE, Robinson HL: Induction of angiosarcoma by c-erbB transducing virus. J Virol 1985, 54:304-310.

20. Hess J, Angel P, Schorpp-Kistner M: AP-1 subunits: quarrel and harmony among siblings. J Cell Sci 2004, 117:5965-5973.

21. Xu X, Wei X, Ling Q, Cheng J, Zhou B, Xie H, Zhou L, Zheng S: Identification of the two portal vein tumor thrombosis associated proteins in hepatocellular carcinoma: protein disulfide-isomerase A6 and apolipoprotein A-I. J Gastroenterol Hepatol 2011, 6:1440.

22. Nicholas Tay, Chan Soh-Ha, EE-Chee R: Identification and cloning of a novel heterogeneous nuclear ribonucleoprotein C-like protein that functions as a transcriptional activator of the Hepatitis B virus enhancer II. J Virol 1992, 66:6841-6848.

23. Yee JK: A liver-specific enhancer in the core promoter region of human hepatitis B virus. Science 1989, 246:658-661.

24. Maas R, Zoelen D, Oei H, Classen I: Replacement of primary chicken embryonic fibroblasts (CEF) by the DF-1 cell line for detection of avian leukosis viruses. Biologicals 2006, 34:177-181.

25. Smith LM, Brown SR, Howes K, Mcleod S, Arshad SS, Barron GS, Venugopal K, Mckay JC, Payne LN: Development and application of polymerase chain reaction(PCR) tests for the detection of subgroup J avian leukosis virus. Virus Res 1998, 54:87-98. 
26. Thompson JD, Gibson TJ, Plewniak F, Jeanmougin F, Higins DG: The Clustal $X$ windows interface: flexible strategies for multiple sequence alignment aided by quality analysis tools. Nucleic Acids Res 1997, 25:4876-4882.

27. Kumar S, Tamura K, Nei M: MEG3: integrated software for molecular evolutionary genetics analysis and sequence alignment. Brief Bioinform 2004, 5:150-163.

28. Zavala G, Cheng S, Jackwood MW: Molecular epidemiology of avian leukosis virus subgroup J and evolutionary history of its $3^{\prime}$ untranslated region. Avian Dis 2007, 51:942-953.

29. Akram A, Dalit SD, Amos P, Amiram E: Avian hemangioma retrovirus induces cell proliferation via the envelope(env) gene. Virology 2000, 276:161-168.

30. Bai J, Howes K, Payne LN, Skinner MA: Sequence of host range determinants in the env gene of a full-length infectious proviral clone of exogenous avian leukosis virus HPRS-103 confirms that it represents a new subgroup (designated J). J Gen Virol 1995, 76:181-187.

31. Chesters PM, Howes K, Petherbridge L, Evans S, Payne LN, Venugopal K: The viral envelope is a major determinant for the induction of lymphoid and myeloid tumours by avian leukosis virus subgroups $\mathrm{A}$ and $\mathrm{J}$, respectively. J Gen Virol 2002, 83:2553-2561.

32. Weiss RA: Cellular receptors and viral glycoproteins involved in retrovirus entry. In In The Retroviridae. Edited by: Levy JA. New York: Plenum; 1992:1-72.

33. Douglas RS, Bjorn V, Michael JH, Hayman J, Paula JE: Nucleotide Sequence of $\mathrm{HBI}$, a novel recombinant MC29 derivative with altered pathogenic properties. J Virol 1985, 56:969-977.

34. Venugopal KK, Howes DM, Flannery MJ: Isolation of acutely transforming subgroup $\mathrm{J}$ avian leucosis viruses that induce erythroblastosis and myelocytomatosis. Avian Pathol 2000, 29:327-332.

35. Ruddell A: Transcription regulatory elements of the avian retroviral long terminal repeat. Virology 1995, 206:1-7.

36. Laimins $L$, Tsichlis $P$, Khoury G: Multiple enhancer domains in the $3^{\prime}$ terminus of the Prague strain of Rous sarcoma virus. Nucleic Acids Res 1984, 12:6427.

37. Bryan RC, Kyle R, Grace JU: Functional analysis of the transcription control region located within the avian retroviral long terminal repeat. Mole Cell Biol 1985, 5:438-447.

38. Shardell S, Lianko G, Ryan C, Michael FS: Potential intra-and intermolecular interactions involving the unique- $5^{\prime}$ region of the HIV-1 $5^{\prime}$ UTR Biochemistry 2008, 47:13064-13073.

39. Magdalena JD, Karen D, Hans EJ, Bruce W, Matthias W, Finn SP: Loss of microRNA targets in the $3^{\prime}$ untranslated region as a mechanism of retroviral insertional activation of growth factor independence. J Virol 2009, 83:8051-8061.

40. Cui ZZ, Zhang Z, Silva RF: Comparison of Chinese field strains of avian leukosis subgroup J viruses with prototype strain HPRS-103 and United States strains. Avian Dis 2003, 47:1321-1330.

41. Schwartz DE, Tizard R, Gilbert F: Nucleotide sequence of Rous sarcoma virus. Cell 1983, 32:853-869.

42. Peter MC, Lorraine PS, Venugopal N: E (XSR) element contributes to the oncogenicity of Avian leukosis virus(subgroup J). J Gen Virol 2006, 87:2685-2692.

43. Guo W, Winistorfer SC, Stoltzfus CM: Selective inhibition of splicing at the avian sarcoma virus src $3^{\prime}$ splice sits by direct-repeat posttranscriptional elements. J Virol 2000, 74:8513-8523.

doi:10.1186/1743-422X-8-552

Cite this article as: Pan et al:: Novel sequences of subgroup J avian leukosis viruses associated with hemangioma in Chinese layer hens.

Virology Journal 2011 8:552.

\section{Submit your next manuscript to BioMed Central and take full advantage of:}

- Convenient online submission

- Thorough peer review

- No space constraints or color figure charges

- Immediate publication on acceptance

- Inclusion in PubMed, CAS, Scopus and Google Scholar

- Research which is freely available for redistribution

Submit your manuscript at www.biomedcentral.com/submit 